\title{
Generalized solutions to boundary value problems for quasilinear hyperbolic systems of partial differential-functional equations
}

\author{
by Tomasz CzŁapiński (Gdańsk)
}

\begin{abstract}
Generalized solutions to quasilinear hyperbolic systems in the second canonical form are investigated. A theorem on existence, uniqueness and continuous dependence upon the boundary data is given. The proof is based on the methods due to L. Cesari and P. Bassanini for systems which are not functional.
\end{abstract}

1. Introduction. We denote by $C(X, Y)$ the set of all continuous functions from $X$ to $Y$, where $X, Y$ are any metric spaces. Let $a_{0}>0$, $B=\left[-b_{0}, 0\right] \times[-b, b]$, where $b_{0} \in \mathbb{R}_{+}, b=\left(b_{1}, \ldots, b_{r}\right) \in \mathbb{R}_{+}^{r}, \mathbb{R}_{+}=[0, \infty)$. For $z:\left[-b_{0}, a_{0}\right] \times \mathbb{R}^{r} \rightarrow \mathbb{R}^{m}$ and $(x, y)=\left(x, y_{1}, \ldots, y_{r}\right) \in\left[0, a_{0}\right] \times \mathbb{R}^{r}$, define $z_{(x, y)}: B \rightarrow \mathbb{R}^{m}$ by $z_{(x, y)}(t, s)=z(x+t, y+s),(t, s) \in B$. Put $\Omega=\left[0, a_{0}\right] \times \mathbb{R}^{r} \times C\left(B, \mathbb{R}^{m}\right)$, and denote by $M(m, r)$ the set of all real $m \times r$ matrices.

Let

$$
\begin{aligned}
A: \Omega \rightarrow M(m, m), & A=\left[A_{i j}\right], i, j=1, \ldots, m, \\
\varrho: \Omega \rightarrow M(m, r), & \varrho=\left[\varrho_{i j}\right], i=1, \ldots, m, j=1, \ldots, r, \\
f: \Omega \rightarrow M(m, 1), & f=\left[f_{1}, \ldots, f_{m}\right]^{T},
\end{aligned}
$$

be given functions of the variables $(x, y, w), y=\left(y_{1}, \ldots, y_{r}\right), w=\left(w_{1}, \ldots, w_{r}\right)$, and $T$ the transpose symbol. Note that if $(x, y) \in\left[0, a_{0}\right] \times \mathbb{R}^{r}$ is fixed then $A(x, y, \cdot), \varrho(x, y, \cdot), f(x, y, \cdot)$ are operators on the function space $C\left(B, \mathbb{R}^{m}\right)$. Furthermore, let

$$
\begin{aligned}
B_{l} & : \mathbb{R}^{r} \rightarrow M(m, m), & B_{l} & =\left[B_{l i j}\right], i, j=1, \ldots, m, \\
\psi & : \mathbb{R}^{r} \rightarrow \mathbb{R}^{r}, & \psi & =\left(\psi_{1}, \ldots, \psi_{r}\right),
\end{aligned}
$$

where $l=1, \ldots, N, m \leq N$, be given functions of the variable $y=\left(y_{1}, \ldots, y_{r}\right)$.

1991 Mathematics Subject Classification: 35L50, 35D05.

Key words and phrases: differential-functional system, second canonical form, generalized solutions. 
We consider the following hyperbolic differential-functional system in the second canonical form:

$$
\text { (1) } \begin{aligned}
\sum_{j=1}^{m} A_{i j}\left(x, y, z_{(x, y)}\right)\left[D_{x} z_{j}(x, y)+\right. & \left.\sum_{k=1}^{r} \varrho_{i k}\left(x, y, z_{(x, y)}\right) D_{y_{k}} z_{j}(x, y)\right] \\
& =f_{i}\left(x, y, z_{(x, y)}\right), \quad i=1, \ldots, m,
\end{aligned}
$$

with the boundary data on $N$ arbitrary (not necessarily distinct) hyperplanes $x=a_{l}, a_{l} \in\left[0, a_{0}\right], l=1, \ldots, N$,

$$
\sum_{l=1}^{N} B_{l}(y) z\left(a_{l}, y\right)=\psi(y), \quad y \in \mathbb{R}^{r} .
$$

A function $z \in C\left(I_{a}, \mathbb{R}^{m}\right), I_{a}=[0, a] \times \mathbb{R}^{r}$, where $a \in\left(0, a_{0}\right]$, is a solution of (1), (2) if $z$ has partial derivatives $D_{x} z, D_{y_{k}} z, k=1, \ldots, r$, a.e. on $I_{a}$, satisfies (1) a.e. on $I_{a}$ and (2) for all $y \in \mathbb{R}^{r}$.

If $b_{0}=0$ and $b=0$ then the differential-functional system (1) reduces to a differential system in the second canonical form. Generalized (a.e.) solutions of systems of that form have been investigated in a large number of papers. We mention here the papers of P. Bassanini [1]-[3] and L. Cesari [5], [6]. Quasilinear hyperbolic systems with a retarded argument have been studied by Z. Kamont and J. Turo [12]-[15]. In [19]-[22] J. Turo has also considered the existence and uniqueness of generalized solutions for differential-functional hyperbolic systems with operators of Volterra type. In that case the given functions are superpositions of functions defined on subsets of a finite-dimensional Euclidean space with operators of Volterra type. The main assumptions for the operators have been formulated in the form of linear inequalities given on some function space. Classical solutions to nonlinear equations with the same model of functional dependence have been investigated in [11], [18].

In this paper we consider a new model of differential-functional systems which has been used in [9] for initial value problems. For each $(x, y) \in$ $\left[-b_{0}, a_{0}\right] \times \mathbb{R}^{r}$ and for any $z:\left[-b_{0}, a_{0}\right] \times \mathbb{R}^{r} \rightarrow \mathbb{R}^{m}$ we denote by $z_{(x, y)}$ the translation of the restriction of $z$ to $B$. In other words, the graph of $z_{(x, y)}$ is the graph of $z:\left[x-b_{0}, x\right] \times[y-b, y+b] \rightarrow \mathbb{R}^{m}$ shifted to $B$. Since the given functions are now operators on $z_{(x, y)}$ we no longer need the assumption that the right-hand side of the system is the superposition of some function and an operator of Volterra type. This simple model is well known for ordinary differential-functional equations (see [10], [16]). It is also very general since systems of differential equations with retarded argument ([12]-[15]), differential-integral systems ([17]) and differential-functional systems with operators of Volterra type ([19]-[22]) can be obtained from (1) by specializing the given functions. Our formulation of the problem enables us to get 
new examples of differential-functional systems which cannot be obtained from the results cited above. More detailed comparison between the model of functional dependence proposed in this paper and that used in [19]-[22] is presented in [7], [8]. The method which we use is based on the Banach fixed point theorem in a product space and it is close to that used in [14].

2. Assumptions and notations. Let $\|\eta\|_{m}=\max _{1 \leq i \leq m}\left|\eta_{i}\right|$ in $\mathbb{R}^{m}$ and $\|U\|_{m, k}=\max _{1 \leq i \leq m} \sum_{j=1}^{m}\left|U_{i j}\right|$ in $M(m, k)$. For short we write $\|\eta\|,\|U\|$. For $w \in C\left(B, \mathbb{R}^{m}\right)$ put $\|w\|_{0}=\sup \{\|w(t, s)\|:(t, s) \in B\}$. Let $C_{0+\mathrm{L}}\left(B, \mathbb{R}^{m}\right)$ denote the set of all functions $w \in C\left(B, \mathbb{R}^{m}\right)$ such that

$\|w\|_{\mathrm{L}}=\sup \left\{[|t-\bar{t}|+\|w-\bar{w}\|]^{-1}\|w(t, s)-w(\bar{t}, \bar{s})\|:(t, s),(\bar{t}, \bar{s}) \in B\right\}<\infty$.

We equip this set with the norm $\|w\|_{0+\mathrm{L}}=\|w\|_{0}+\|w\|_{\mathrm{L}}, w \in C_{0+\mathrm{L}}\left(B, \mathbb{R}^{m}\right)$. Furthermore, for any $q \in \mathbb{R}_{+}$we set $C\left(B, \mathbb{R}^{m}, q\right)=\left\{w \in C\left(B, \mathbb{R}^{m}\right):\|w\|_{0} \leq\right.$ $q\}, C_{0+\mathrm{L}}\left(B, \mathbb{R}^{m}, q\right)=\left\{w \in C_{0+\mathrm{L}}\left(B, \mathbb{R}^{m}\right):\|w\|_{0+\mathrm{L}} \leq q\right\}$.

Assumption $\mathrm{H}_{1} \cdot 1^{\mathrm{o}} A \in C(\Omega, M(m, m))$ and there is a constant $\nu>0$ such that $\operatorname{det} A(x, y, w) \geq \nu$ for any $(x, y, w) \in \Omega$.

$2^{\mathrm{o}}$ There are nondecreasing functions $n, l: \mathbb{R}_{+} \rightarrow \mathbb{R}_{+}$such that for any $q \in \mathbb{R}_{+}$we have

(i) $\|A(x, y, w)\| \leq n(q),(x, y, w) \in\left[0, a_{0}\right] \times \mathbb{R}^{r} \times C\left(B, \mathbb{R}^{m}, q\right)$,

(ii) $\|A(x, y, w)-A(\bar{x}, \bar{y}, \bar{w})\| \leq l(q)\left[|x-\bar{x}|+\|y-\bar{y}\|+\|w-\bar{w}\|_{0}\right]$, $(x, y, w),(\bar{x}, \bar{y}, \bar{w}) \in\left[0, a_{0}\right] \times \mathbb{R}^{r} \times C_{0+\mathrm{L}}\left(B, \mathbb{R}^{m}, q\right)$.

Remark 1. Assumption $\mathrm{H}_{1}$ yields that $A^{-1}(x, y, w)$ exists for any $(x, y, w) \in \Omega$. Furthermore, there are nondecreasing $n^{\prime}, l^{\prime}: \mathbb{R}_{+} \rightarrow \mathbb{R}_{+}$such that condition $2^{\circ}$ of Assumption $\mathrm{H}_{1}$ holds with $A, n, l$ replaced by $A^{-1}, n^{\prime}, l^{\prime}$, respectively.

Let $\theta$ denote the set of all functions $l:\left[0, a_{0}\right] \times \mathbb{R}_{+} \rightarrow \mathbb{R}_{+}$such that $l(\cdot, q):\left[0, a_{0}\right] \rightarrow \mathbb{R}_{+}$is measurable for any $q \in \mathbb{R}_{+}$, and $l(x, \cdot): \mathbb{R}_{+} \rightarrow \mathbb{R}_{+}$is nondecreasing for a.e. $x \in\left[0, a_{0}\right]$.

Assumption $\mathrm{H}_{2} \cdot 1^{\mathrm{o}} \varrho(\cdot, y, w):\left[0, a_{0}\right] \rightarrow M(m, r)$ is measurable for all $(y, w) \in \mathbb{R}^{r} \times C\left(B, \mathbb{R}^{m}\right)$.

$2^{\text {o }} \varrho(x, \cdot): \mathbb{R}^{r} \times C\left(B, \mathbb{R}^{m}\right) \rightarrow M(m, r)$ is continuous for a.e. $x \in\left[0, a_{0}\right]$.

$3^{\text {o }}$ There are a nondecreasing function $n_{1}: \mathbb{R}_{+} \rightarrow \mathbb{R}_{+}$and $l_{1} \in \theta$ such that for any $q \in \mathbb{R}_{+}$we have

(i) $\|\varrho(x, y, w)\| \leq n_{1}(q),(x, y, w) \in\left[0, a_{0}\right] \times \mathbb{R}^{r} \times C\left(B, \mathbb{R}^{m}, q\right)$,

(ii) $\|\varrho(x, y, w)-\varrho(x, \bar{y}, \bar{w})\| \leq l_{1}(x, q)\left[\|y-\bar{y}\|+\|w-\bar{w}\|_{0}\right]$ for all $(y, w)$, $(\bar{y}, \bar{w}) \in \mathbb{R}^{r} \times C_{0+\mathrm{L}}\left(B, \mathbb{R}^{m}, q\right)$ and for a.e. $x \in\left[0, a_{0}\right]$.

Assumption $\mathrm{H}_{3}$. $1^{\mathrm{o}} f(\cdot, y, w):\left[0, a_{0}\right] \rightarrow M(m, 1)$ is measurable for all $(y, w) \in \mathbb{R}^{r} \times C\left(B, \mathbb{R}^{m}\right)$.

$2^{\mathrm{o}} f(x, \cdot): \mathbb{R}^{r} \times C\left(B, \mathbb{R}^{m}\right) \rightarrow M(m, 1)$ is continuous for a.e. $x \in\left[0, a_{0}\right]$. 
$3^{\circ}$ There are a nondecreasing function $n_{2}: \mathbb{R}_{+} \rightarrow \mathbb{R}_{+}$and $l_{2} \in \theta$ such that for any $q \in \mathbb{R}_{+}$we have

(i) $\|f(x, y, w)\| \leq n_{2}(q),(x, y, w) \in\left[0, a_{0}\right] \times \mathbb{R}^{r} \times C\left(B, \mathbb{R}^{m}, q\right)$,

(ii) $\|f(x, y, w)-f(x, \bar{y}, \bar{w})\| \leq l_{2}(x, q)\left[\|y-\bar{y}\|+\|w-\bar{w}\|_{0}\right]$ for all $(y, w)$, $(\bar{y}, \bar{w}) \in \mathbb{R}^{r} \times C_{0+\mathrm{L}}\left(B, \mathbb{R}^{m}, q\right)$ and for a.e. $x \in\left[0, a_{0}\right]$.

For $(x, y, w) \in \Omega, l=1, \ldots, N$, we put

$$
\begin{gathered}
A(x, y, w)=E+\widetilde{A}(x, y, w), \quad A^{-1}(x, y, w)=E+\bar{A}(x, y, w), \\
B_{l}(y)=E_{l}+\widetilde{B}_{l}(y), \quad E=\left[\delta_{i j}\right], \quad E_{l}=\left[\delta_{l i} \delta_{i j}\right], \quad i, j=1, \ldots, m,
\end{gathered}
$$

and

$$
\begin{aligned}
& \sigma_{0}=\sup \left\{\sum_{l=1}^{N}\left\|\widetilde{B}_{l}(y)\right\|: y \in \mathbb{R}^{r}\right\}, \\
& \sigma_{1}=\sup \{\|\widetilde{A}(x, y, w)\|:(x, y, w) \in \Omega\}, \\
& \sigma_{2}=\sup \{\|\bar{A}(x, y, w)\|:(x, y, w) \in \Omega\} .
\end{aligned}
$$

Assumption $\mathrm{H}_{4} \cdot 1^{\circ} \psi \in C\left(\mathbb{R}^{r}, \mathbb{R}^{m}\right)$ and there are constants $\Gamma, \Lambda \in \mathbb{R}_{+}$ such that for all $y, \bar{y} \in \mathbb{R}^{r}$ we have

$$
\|\psi(y)\| \leq \Gamma, \quad\|\psi(y)-\psi(\bar{y})\| \leq \Lambda\|y-\bar{y}\| .
$$

$2^{\circ} B_{l} \in C\left(\mathbb{R}^{r}, M(m, m)\right)$, $\operatorname{det} B_{l}(y) \neq 0$ for all $l=1, \ldots, N, y \in \mathbb{R}^{r}$, and there is a constant $G \in \mathbb{R}_{+}$such that

$$
\sum_{l=1}^{N}\left\|B_{l}(y)-B_{l}(\bar{y})\right\| \leq G\|y-\bar{y}\|, \quad y, \bar{y} \in \mathbb{R}^{r} .
$$

$3^{\circ}\left(\sigma_{0}+\sigma_{1}\right)\left(1+\sigma_{2}\right)<1$.

We denote by $B_{1}\left(a, Q, Q_{1}, Q_{2}\right)$, where $a \in\left(0, a_{0}\right], Q, Q_{1}, Q_{2} \in \mathbb{R}_{+}$, the set of all functions $z \in C\left(I_{a}, \mathbb{R}^{m}\right)$ such that for all $(x, y),(\bar{x}, \bar{y}) \in I_{a}$ we have

(i) $\|z(x, y)\| \leq Q$,

(ii) $\|z(x, y)-z(\bar{x}, \bar{y})\| \leq Q_{1}|x-\bar{x}|+Q_{2}\|y-\bar{y}\|$.

Note that $B_{1}\left(a, Q, Q_{1}, Q_{2}\right)$ is a closed subset of the Banach space of all continuous and bounded vector functions $z: I_{a} \rightarrow \mathbb{R}^{m}$ with the norm $\|z\|_{I_{a}}=\sup \left\{\|z(x, y)\|:(x, y) \in I_{a}\right\}$.

For $a \in\left(0, a_{0}\right]$ define the constants $L_{i a}\left(Q, Q_{1}, Q_{2}\right)=\int_{0}^{a} l_{i}\left(t, Q+Q_{1}\right.$ $\left.+Q_{2}\right) d t, i=1,2$. In the sequel we write $L_{i a}$ for short.

Let $a \in\left(0, a_{0}\right]$ be so small that

$$
L_{1 a}\left(1+Q_{2}\right)<1
$$

and let $Q, Q_{1}, Q_{2} \in \mathbb{R}_{+}, p \in(0,1)$. Then we denote by $B_{2}\left(a, Q, Q_{1}, Q_{2}, p\right)$ the set of all functions $h \in C\left(\Delta_{a}, M(m, r)\right), \Delta_{a}=[0, a] \times[0, a] \times \mathbb{R}^{r}$, such that for all $(x, x, y),(\xi, x, y),(\bar{\xi}, \bar{x}, \bar{y}) \in \Delta_{a}, i=1, \ldots, m$, we have 
(i) $h_{i}(x, x, y)=0$,

(ii) $\left\|h_{i}(\xi, x, y)-h_{i}(\bar{\xi}, \bar{x}, \bar{y})\right\| \leq n_{1}(Q)|\xi-\bar{\xi}|+\gamma_{a} n_{1}(Q)|x-\bar{x}|+p\|y-\bar{y}\|$, where $\gamma_{a}=\left[1-L_{1 a}\left(1+Q_{2}\right)\right]^{-1}, h_{i}=\left(h_{i 1}, \ldots, h_{i r}\right)$. It is easily seen that $B_{2}\left(a, Q, Q_{1}, Q_{2}, p\right)$ is a closed subset of the Banach space of all continuous and bounded matrix functions $h: \Delta_{a} \rightarrow M(m, r)$ with the norm $\|h\|_{\Delta_{a}}=$ $\max _{1 \leq i \leq m} \sup \left\{\left\|h_{i}(\xi, x, y)\right\|:(\xi, x, y) \in \Delta_{a}\right\}$. Indeed, for all $(\xi, x, y) \in \Delta_{a}$, $i=1, \ldots, m$, we have

$$
\left\|h_{i}(\xi, x, y)\right\|=\left\|h_{i}(\xi, x, y)-h_{i}(x, x, y)\right\| \leq n_{1}(Q)|\xi-x| \leq n_{1}(Q) a,
$$

which yields $\|h\|_{\Delta_{a}} \leq n_{1}(Q) a$.

For any $h \in B_{2}\left(a, Q, Q_{1}, Q_{2}, p\right)$ let $g: \Delta_{a} \rightarrow M(m, r)$ be defined by

$$
g_{i}(\xi, x, y)=y+h_{i}(\xi, x, y), \quad(\xi, x, y) \in \Delta_{a}, i=1, \ldots, m,
$$

where $g_{i}=\left(g_{i 1}, \ldots, g_{i r}\right), h_{i}=\left(h_{i 1}, \ldots, h_{i r}\right)$. Then for all $(x, x, y),(\xi, x, y)$, $(\bar{\xi}, \bar{x}, \bar{y}) \in \Delta_{a}, i=1, \ldots, m$, we have

(i) $g_{i}(x, x, y)=y$,

(ii) $\left\|g_{i}(\xi, x, y)-g_{i}(\bar{\xi}, \bar{x}, \bar{y})\right\| \leq n_{1}(Q)|\xi-\bar{\xi}|+\gamma_{a} n_{1}(Q)|x-\bar{x}|+(1+p)\|y-\bar{y}\|$.

3. The operators $V^{(1)}, V^{(2)}$ and their properties. Let $\langle\cdot, \cdot\rangle$ be a scalar product in $\mathbb{R}^{m}$, let $g_{i}(t, x, y)$ be defined by (5) and set

$$
\begin{aligned}
& z_{i}^{*}(t, x, y)=\left(z_{1}\left(t, g_{i}(t, x, y)\right), \ldots, z_{m}\left(t, g_{i}(t, x, y)\right)\right), \\
& A_{i}^{*}(t, x, y) \\
& \quad=\left(A_{i 1}\left(t, g_{i}(t, x, y), z_{\left(t, g_{i}(t, x, y)\right)}\right), \ldots, A_{i m}\left(t, g_{i}(t, x, y), z_{\left(t, g_{i}(t, x, y)\right)}\right)\right) .
\end{aligned}
$$

We consider the operators $Z=V^{(1)}[z, h], H=V^{(2)}[z, h]$ defined for $z \in$ $B_{1}\left(a, Q, Q_{1}, Q_{2}\right), h \in B_{2}\left(a, Q, Q_{1}, Q_{2}, p\right)$ as follows:

$$
\begin{aligned}
Z(x, y) & =A^{-1}\left(x, y, z_{(x, y)}\right)\left(\Delta_{1}(x, y)+\Delta_{2}(x, y)+\Delta_{3}(x, y)\right), \\
\Delta_{k}(x, y) & =\left(\Delta_{k 1}(x, y), \ldots, \Delta_{k m}(x, y)\right), \quad k=1,2,3, \\
\Delta_{1 i}(x, y) & =\psi_{i}\left(g_{i}\left(a_{i}, x, y\right)\right)+\int_{a_{i}}^{x} f_{i}\left(t, g_{i}(t, x, y), z_{\left(t, g_{i}(t, x, y)\right)}\right) d t,
\end{aligned}
$$

(6) $\quad \Delta_{2 i}(x, y)=\int_{a_{i}}^{x}\left\langle D_{t} A_{i}^{*}(t, x, y), z_{i}^{*}(t, x, y)\right\rangle d t$

$$
\begin{array}{r}
+\left\langle A_{i}^{*}\left(a_{i}, x, y\right), z_{i}^{*}\left(a_{i}, x, y\right)\right\rangle, \\
\Delta_{3 i}(x, y)=-\sum_{l=1}^{N}\left\langle B_{l i}\left(g_{i}\left(a_{i}, x, y\right)\right), z\left(a_{l}, g_{i}\left(a_{i}, x, y\right)\right)\right\rangle, \\
i=1, \ldots, m, \quad(x, y) \in I_{a} ;
\end{array}
$$


and

$$
\begin{aligned}
& H(\xi, x, y)=\left[H_{i j}(\xi, x, y)\right], \quad i=1, \ldots, m, j=1, \ldots, r, \\
& H_{i}(\xi, x, y)=\int_{x}^{\xi} \varrho_{i}\left(t, g_{i}(t, x, y), z_{\left(t, g_{i}(t, x, y)\right)}\right) d t \\
& i=1, \ldots, m,(\xi, x, y) \in \Delta_{a} .
\end{aligned}
$$

Remark 2. By (3) we may simultaneously replace $D_{t} A_{i}^{*}, A_{i}, B_{l i}$ in the above equalities by $D_{t} \widetilde{A}_{i}^{*}, \widetilde{A}_{i}, \widetilde{B}_{l i}$, respectively.

Lemma 1. Suppose that Assumptions $\mathrm{H}_{1}-\mathrm{H}_{4}$ hold and that

(8) $\left(1+\sigma_{2}\right) \Gamma+\left(1+\sigma_{2}\right)\left(\sigma_{0}+\sigma_{1}\right) Q<Q$,

(9) $l^{\prime}(Q)\left(1+Q_{1}\right)\left[\Gamma+\left(\sigma_{0}+\sigma_{1}\right) Q\right]+\left(1+\sigma_{2}\right)\left[n_{1}(Q)(\Lambda+G Q)\right.$

$$
\begin{aligned}
+l(Q) Q(2 & \left.\left.+n_{1}(Q)+2 Q_{1}+Q_{2} n_{1}(Q)\right)\right] \\
& +n_{1}(Q)\left(1+\sigma_{2}\right)\left(\sigma_{0}+\sigma_{1}\right) Q_{2}<Q_{1},
\end{aligned}
$$

(10) $l^{\prime}(Q)\left(1+Q_{2}\right)\left[\Gamma+\left(\sigma_{0}+\sigma_{1}\right) Q\right]+\left(1+\sigma_{2}\right)\left[(1+p)(\Lambda+G Q)+l(Q) Q\left(1+Q_{2}\right)\right]$

$$
+(1+p)\left(1+\sigma_{2}\right)\left(\sigma_{0}+\sigma_{1}\right) Q_{2}<Q_{2} .
$$

Then for $a \in\left(0, a_{0}\right]$ sufficiently small the operator $V^{(1)}$ maps $B_{1}\left(a, Q, Q_{1}, Q_{2}\right)$ $\times B_{2}\left(a, Q, Q_{1}, Q_{2}, p\right)$ into $B_{1}\left(a, Q, Q_{1}, Q_{2}\right)$.

Proof. Let $Z=V^{(1)}[z, h], z \in B_{1}\left(a, Q, Q_{1}, Q_{2}\right), h \in B_{2}\left(a, Q, Q_{1}, Q_{2}, p\right)$. By the estimates

$$
\begin{aligned}
\left\|\Delta_{1}(x, y)\right\| \leq & \max _{1 \leq i \leq m}\left\{\left|\psi_{i}\left(g_{i}\left(a_{i}, x, y\right)\right)\right|\right. \\
& \left.+\left|\int_{a_{i}}^{x} f_{i}\left(t, g_{i}(t, x, y), z_{\left(t, g_{i}(t, x, y)\right)}\right) d t\right|\right\} \\
\leq & \Gamma+\int_{a_{i}}^{x} n_{2}(Q) d t \leq \Gamma+n_{2}(Q) a, \\
\left\|\Delta_{2}(x, y)\right\| \leq & \max _{1 \leq i \leq m}\left\{\left|\int_{a_{i}}^{x}\left\langle D_{t} \widetilde{A}_{i}^{*}(t, x, y), z_{i}^{*}(t, x, y)\right\rangle d t\right|\right. \\
& \left.+\left|\left\langle\widetilde{A}_{i}^{*}\left(a_{i}, x, y\right), z_{i}^{*}\left(a_{i}, x, y\right)\right\rangle\right|\right\} \\
\leq & \int_{a_{i}}^{x} l(Q)\left(1+n_{1}(Q)+Q_{1}+Q_{2} n_{1}(Q)\right) Q d t+\sigma_{1} Q \\
\leq & l(Q)\left(1+n_{1}(Q)+Q_{1}+Q_{2} n_{1}(Q)\right) Q a+\sigma_{1} Q, \\
\left\|\Delta_{3}(x, y)\right\| \leq & \max _{1 \leq i \leq m}\left|-\sum_{l=1}^{N}\left\langle\widetilde{B}_{l i}\left(g_{i}\left(a_{i}, x, y\right)\right), z\left(a_{l}, g_{i}\left(a_{i}, x, y\right)\right)\right\rangle\right| \leq \sigma_{0} Q,
\end{aligned}
$$


we obtain

$\|Z(x, y)\|$

$\leq\left(1+\sigma_{2}\right)\left[\Gamma+n_{2}(Q) a+l(Q)\left(1+n_{1}(Q)+Q_{1}+Q_{2} n_{1}(Q)\right) Q a+\sigma_{1} Q+\sigma_{0} Q\right]$,

$(x, y) \in I_{a}$. By (8) we can choose $a$ so small that

(11) $\quad\left(1+\sigma_{2}\right)\left[\Gamma+\left(\sigma_{1}+\sigma_{0}\right) Q\right]$

$$
+\left(1+\sigma_{2}\right)\left[n_{2}(Q)+l(Q)\left(1+n_{1}(Q)+Q_{1}+Q_{2} n_{1}(Q)\right) Q\right] a \leq Q,
$$

and thus for $(x, y) \in I_{a}$ we obtain

$$
\|Z(x, y)\| \leq Q .
$$

For any $(x, y),(\bar{x}, \bar{y}) \in I_{a}$, we have

$$
Z(x, y)-Z(\bar{x}, \bar{y})=\alpha_{0}+\alpha_{1}+\alpha_{2}+\alpha_{3},
$$

where

$$
\begin{aligned}
& \alpha_{0}=\left(A^{-1}\left(x, y, z_{(x, y)}\right)-A^{-1}\left(\bar{x}, \bar{y}, z_{(\bar{x}, \bar{y})}\right)\left[\Delta_{1}(x, y)+\Delta_{2}(x, y)+\Delta_{3}(x, y)\right],\right. \\
& \alpha_{i}=A^{-1}\left(\bar{x}, \bar{y}, z_{(\bar{x}, \bar{y})}\right)\left[\Delta_{i}(x, y)-\Delta_{i}(\bar{x}, \bar{y})\right], \quad i=1,2,3 .
\end{aligned}
$$

We can estimate the above terms as follows:

$$
\begin{aligned}
& \left\|\alpha_{0}\right\| \leq\left[l^{\prime}(Q)\left(1+Q_{2}\right)|x-\bar{x}|+l^{\prime}(Q)\left(1+Q_{2}\right)\|y-\bar{y}\|\right] \\
& \times\left[\left\|\Delta_{1}(x, y)\right\|+\left\|\Delta_{2}(x, y)\right\|+\left\|\Delta_{3}(x, y)\right\|\right] \\
& \leq\left[\Gamma+n_{2}(Q) a+l(Q)\left(1+n_{1}(Q)+Q_{1}+Q_{2} n_{1}(Q)\right) Q a\right. \\
& \left.+\sigma_{1} Q+\sigma_{0} Q\right] l^{\prime}(Q)\left(1+Q_{1}\right)|x-\bar{x}| \\
& +\left[\Gamma+n_{2}(Q) a+l(Q)\left(1+n_{1}(Q)+Q_{1}+Q_{2} n_{1}(Q)\right) Q a\right. \\
& \left.+\sigma_{1} Q+\sigma_{0} Q\right] l^{\prime}(Q)\left(1+Q_{1}\right)\|y-\bar{y}\|, \\
& \left\|\alpha_{1}\right\| \leq\left(1+\sigma_{2}\right) \max _{1 \leq i \leq m}\left\{\left|\psi_{i}\left(g_{i}\left(a_{i}, x, y\right)\right)-\psi_{i}\left(g_{i}\left(a_{i}, \bar{x}, \bar{y}\right)\right)\right|\right. \\
& +\mid \int_{a_{i}}^{x}\left[f_{i}\left(t, g_{i}(t, x, y), z_{\left(t, g_{i}(t, x, y)\right)}\right)\right. \\
& \left.-f_{i}\left(t, g_{i}(t, \bar{x}, \bar{y}), z_{\left(t, g_{i}(t, \bar{x}, \bar{y})\right)}\right)\right] d t \\
& \left.+\left|\int_{\bar{x}}^{x} f_{i}\left(t, g_{i}(t, \bar{x}, \bar{y}), z_{\left(t, g_{i}(t, \bar{x}, \bar{y})\right)}\right) d t\right|\right\} \\
& \leq\left(1+\sigma_{2}\right)\left[\Lambda \gamma_{a}+L_{2 a}\left(1+Q_{2}\right) \gamma_{a}+n_{2}(Q) a\right]|x-\bar{x}| \\
& +\left(1+\sigma_{2}\right)\left[\Lambda(1+p)+L_{2 a}\left(1+Q_{2}\right)(1+p)\right]\|y-\bar{y}\|, \\
& \left\|\alpha_{2}\right\| \leq\left(1+\sigma_{2}\right) \max _{1 \leq i \leq m}\left\{\left|\int_{a_{i}}^{x}\left\langle\widetilde{A}_{i}^{*}(t, x, y)-\widetilde{A}_{i}^{*}(t, \bar{x}, \bar{y}), D_{t} z_{i}^{*}(t, x, y)\right\rangle d t\right|\right.
\end{aligned}
$$




$$
\begin{aligned}
&++\int_{a_{i}}^{x}\left\langle D_{t} \widetilde{A}_{i}^{*}(t, \bar{x}, \bar{y}), z_{i}^{*}(t, x, y)-z_{i}^{*}(t, \bar{x}, \bar{y})\right\rangle d t \mid \\
&+\left|\int_{\bar{x}}^{x}\left\langle D_{t} \widetilde{A}_{i}^{*}(t, \bar{x}, \bar{y}), z_{i}^{*}(t, x, y)\right\rangle d t\right| \\
&+\left|\left\langle\widetilde{A}_{i}^{*}(x, x, y)-\widetilde{A}_{i}^{*}(\bar{x}, \bar{x}, \bar{y}), z_{i}^{*}(x, x, y)\right\rangle\right| \\
&\left.+\left|\left\langle\widetilde{A}_{i}^{*}\left(a_{i}, \bar{x}, \bar{y}\right), z_{i}^{*}\left(a_{i}, x, y\right)-z_{i}^{*}\left(a_{i}, \bar{x}, \bar{y}\right)\right\rangle\right|\right\} \\
& \leq\left(1+\sigma_{2}\right)\left\{l(Q)\left(1+Q_{2}\right) \gamma_{a}\left(Q_{1}+Q_{2} n_{1}(Q)\right) a\right. \\
&+l(Q)\left(1+n_{1}(Q)+Q_{1}+Q_{2} n_{1}(Q)\right) Q_{2} \gamma_{a} a \\
&+l(Q)\left(1+n_{1}(Q)+Q_{1}+Q_{2} n_{1}(Q)\right) Q \\
&\left.+l(Q)\left(1+Q_{1}\right) Q+\sigma_{1} Q_{2} \gamma_{a}\right\}|x-\bar{x}| \\
&+\left(1+\sigma_{2}\right)\left\{l(Q)\left(1+Q_{2}\right)(1+p)\left(Q_{1}+Q_{2} n_{1}(Q)\right) a\right. \\
&+l(Q)\left(1+n_{1}(Q)+Q_{1}+Q_{2} n_{1}(Q)\right) Q_{2}(1+p) a \\
&\left.+l(Q)\left(1+Q_{2}\right) Q+\sigma_{1} Q_{2}(1+p)\right\}\|y-\bar{y}\|,
\end{aligned}
$$

$\left\|\alpha_{3}\right\| \leq\left(1+\sigma_{2}\right)$

$$
\begin{aligned}
& \times \max _{1 \leq i \leq m}\left\{\left|\sum_{l=1}^{N}\left\langle\widetilde{B}_{l i}\left(g_{i}\left(a_{i}, x, y\right)\right)-\widetilde{B}_{l i}\left(g_{i}\left(a_{i}, \bar{x}, \bar{y}\right)\right), z\left(a_{l}, g_{i}\left(a_{i}, x, y\right)\right)\right\rangle\right|\right. \\
& \left.+\left|\sum_{l=1}^{N}\left\langle\widetilde{B}_{l i}\left(g_{i}\left(a_{i}, \bar{x}, \bar{y}\right)\right), z\left(a_{l}, g_{i}\left(a_{i}, x, y\right)\right)-z\left(a_{l}, g_{i}\left(a_{i}, \bar{x}, \bar{y}\right)\right)\right\rangle\right|\right\} \\
\leq & \left(1+\sigma_{2}\right) \gamma_{a}\left[G Q+\sigma_{0} Q_{2}\right]|x-\bar{x}|+\left(1+\sigma_{2}\right)(1+p)\left[G Q+\sigma_{0} Q_{2}\right]\|y-\bar{y}\| .
\end{aligned}
$$

In estimating $\alpha_{2}$ we have used integration by parts. From the above inequalities we obtain

$$
\|Z(x, y)-Z(\bar{x}, \bar{y})\| \leq W_{1 a}|x-\bar{x}|+W_{2 a}\|y-\bar{y}\|,
$$

where

$$
\begin{aligned}
W_{1 a}= & l^{\prime}(Q)\left(1+Q_{1}\right)\left[\Gamma+\left(\sigma_{1}+\sigma_{0}\right)\right] \\
& +\left(1+\sigma_{2}\right)\left[\gamma_{a}(\Lambda+G Q)+l(Q) Q\left(2+n_{1}(Q)+2 Q_{1}+Q_{2} n_{1}(Q)\right)\right] \\
& +\gamma_{a}\left(1+\sigma_{2}\right)\left(\sigma_{1}+\sigma_{0}\right) Q_{2}+W_{1 a}^{\prime}, \\
W_{2 a}= & l^{\prime}(Q)\left(1+Q_{2}\right)\left[\Gamma+\left(\sigma_{1}+\sigma_{0}\right)\right] \\
& +\left(1+\sigma_{2}\right)\left[(1+p)(\Lambda+G Q)+l(Q) Q\left(1+Q_{2}\right)\right] \\
& +(1+p)\left(1+\sigma_{2}\right)\left(\sigma_{1}+\sigma_{0}\right) Q_{2}+W_{2 a}^{\prime},
\end{aligned}
$$

and $W_{1 a}^{\prime} \geq 0, W_{2 a}^{\prime} \geq 0$ are some constants such that $\lim _{a \rightarrow 0^{+}} W_{1 a}^{\prime}=$ $\lim _{a \rightarrow 0^{+}} W_{2 a}^{\prime}=0$. By (9), (10) we can choose $a$ so small that

$$
W_{1 a} \leq Q_{1}, \quad W_{2 a} \leq Q_{2} .
$$


Hence, for any $(x, y),(\bar{x}, \bar{y}) \in I_{a}$ we get

$$
\|Z(x, y)-Z(\bar{x}, \bar{y})\| \leq Q_{1}|x-\bar{x}|+Q_{2}\|y-\bar{y}\| .
$$

Finally, if $a \in\left(0, a_{0}\right]$ is so small that inequalities (4), (11), (13) hold, then by (12), (14) we have $Z \in B_{1}\left(a, Q, Q_{1}, Q_{2}\right)$. This completes the proof.

Lemma 2. Suppose that Assumption $\mathrm{H}_{2}$ holds, $Q, Q_{1}, Q_{2} \in \mathbb{R}_{+}, p \in(0,1)$ and $a \in\left(0, a_{0}\right]$ is so small that

$$
L_{1 a}(1+p)(1+Q) \leq p .
$$

Then the operator $V^{(2)}$ maps $B_{1}\left(a, Q, Q_{1}, Q_{2}\right) \times B_{2}\left(a, Q, Q_{1}, Q_{2}, p\right)$ into $B_{2}\left(a, Q, Q_{1}, Q_{2}\right)$.

Proof. Let $H=V^{(2)}[z, h], z \in B_{1}\left(a, Q, Q_{1}, Q_{2}\right), h \in B_{2}\left(a, Q, Q_{1}, Q_{2}, p\right)$. It is easy to see that for any $(\xi, x, y),(\bar{\xi}, x, y),(\xi, x, \bar{y}) \in \Delta_{a}, i=1, \ldots, m$, we have

$$
\begin{gathered}
H_{i}(x, x, y)=0, \quad\left\|H_{i}(\xi, x, y)-H_{i}(\bar{\xi}, x, y)\right\| \leq n_{1}(Q)|\xi-\bar{\xi}|, \\
\left\|H_{i}(\xi, x, y)-H_{i}(\xi, x, \bar{y})\right\| \\
\leq\left|\int_{x}^{\xi}\left\|\varrho_{i}\left(t, g_{i}(t, x, y), z_{\left(t, g_{i}(t, x, y)\right)}\right)-\varrho_{i}\left(t, g_{i}(t, x, \bar{y}), z_{\left(t, g_{i}(t, x, \bar{y})\right)}\right)\right\| d t\right| \\
\leq\left|\int_{x}^{\xi} l_{1}(t, P+Q)(1+Q)\left\|g_{i}(t, x, y)-g_{i}(t, x, \bar{y})\right\| d t\right| \\
\leq L_{1 a}(1+Q)(1+p)\|y-\bar{y}\| \leq p\|y-\bar{y}\|
\end{gathered}
$$

by (15). Note that (15) implies (4), and thus for any $(\xi, x, y),(\xi, \bar{x}, y) \in \Delta_{a}$, $i=1, \ldots, m$, we have

$$
\begin{aligned}
& \left\|H_{i}(\xi, x, y)-H_{i}(\xi, \bar{x}, y)\right\| \leq\left|\int_{x}^{\bar{x}}\left\|\varrho_{i}\left(t, g_{i}(t, \bar{x}, y), z_{\left(t, g_{i}(t, \bar{x}, y)\right)}\right)\right\| d t\right| \\
& \quad+\left|\int_{x}^{\xi}\left\|\varrho_{i}\left(t, g_{i}(t, x, y), z_{\left(t, g_{i}(t, x, y)\right)}\right)-\varrho_{i}\left(t, g_{i}(t, \bar{x}, y), z_{\left(t, g_{i}(t, \bar{x}, y)\right)}\right)\right\| d t\right| \\
& \leq\left[n_{1}(Q)+L_{1 a}(1+Q) \gamma_{a}\right]|x-\bar{x}|=\gamma_{a}|x-\bar{x}| .
\end{aligned}
$$

From the above inequalities we derive $H \in B_{2}\left(a, Q, Q_{1}, Q_{2}, p\right)$. This is our claim.

For $a \in\left(0, a_{0}\right]$ define constants

$$
\begin{aligned}
E_{1 a}= & l^{\prime}(Q)\left[\Gamma+n_{2}(Q) a+l(Q)\left(1+n_{1}(Q)+Q_{1}+Q_{2} n_{1}(Q)\right) Q a+\sigma_{1} Q\right. \\
& \left.+\sigma_{0} Q\right]+\left(1+\sigma_{2}\right)\left[L_{2 a}+l(Q)\left(Q_{1}+Q_{2} n_{1}(Q)\right) a\right. \\
& \left.+l(Q)\left(1+n_{1}(Q)+Q_{1}+Q_{2} n_{1}(Q)\right) a+l(Q) Q+\sigma_{1}+\sigma_{0}\right],
\end{aligned}
$$




$$
\begin{aligned}
E_{2 a}= & \left(1+\sigma_{2}\right)\left[\Lambda+L_{2 a}\left(1+Q_{2}\right)+l(Q)\left(1+Q_{2}\right)\left(Q_{1}+Q_{2} n_{1}(Q)\right) a\right. \\
& \left.+l(Q) Q_{1}\left(1+n_{1}(Q)+Q_{1}+Q_{2} n_{1}(Q)\right) a+\sigma_{1} Q_{2}+G Q+\sigma_{0} Q_{2}\right] .
\end{aligned}
$$

Lemma 3. Let Assumptions $\mathrm{H}_{1}-\mathrm{H}_{4}$ hold, $Q, Q_{1}, Q_{2} \in \mathbb{R}_{+}, p \in(0,1)$ and $a \in\left(0, a_{0}\right]$ be so small that inequality (4) holds. Then for any $z, z^{\prime} \in$ $B_{1}\left(a, Q, Q_{1}, Q_{2}\right), h, h^{\prime} \in B_{2}\left(a, Q, Q_{1}, Q_{2}, p\right)$, we have

(16) $\left\|V^{(1)}[z, h]-V^{(1)}\left[z^{\prime}, h^{\prime}\right]\right\|_{I_{a}} \leq E_{1 a}\left\|z-z^{\prime}\right\|_{I_{a}}+E_{2 a}\left\|h-h^{\prime}\right\|_{\Delta_{a}}$,

(17) $\left\|V^{(2)}[z, h]-V^{(2)}\left[z^{\prime}, h^{\prime}\right]\right\|_{I_{a}} \leq L_{1 a}\left\|z-z^{\prime}\right\|_{I_{a}}+L_{1 a}(1+Q)\left\|h-h^{\prime}\right\|_{\Delta_{a}}$.

Proof. Let $z, z^{\prime} \in B_{1}\left(a, Q, Q_{1}, Q_{2}\right)$ and $h, h^{\prime} \in B_{2}\left(a, Q, Q_{1}, Q_{2}, p\right)$. For any $(x, y) \in I_{a}$ we have

$$
V^{(1)}[z, h](x, y)-V^{(1)}\left[z^{\prime}, h^{\prime}\right](x, y)=\beta_{0}+\beta_{1}+\beta_{2}+\beta_{3},
$$

where

$$
\begin{aligned}
& \beta_{0}=\left(A^{-1}\left(x, y, z_{(x, y)}\right)-A^{-1}\left(x, y, z_{(x, y)}^{\prime}\right)\right)\left[\Delta_{1}(x, y)+\Delta_{2}(x, y)+\Delta_{3}(x, y)\right], \\
& \beta_{i}=A^{-1}\left(x, y, z_{(x, y)}^{\prime}\right)\left[\Delta_{i}(x, y)-\Delta_{i}^{\prime}(x, y)\right], \quad i=1,2,3,
\end{aligned}
$$

and formulas for $\Delta_{1}^{\prime}, \Delta_{2}^{\prime}, \Delta_{3}^{\prime}$ arise from (6) by replacing $z, h$ by $z^{\prime}, h^{\prime}$, respectively. Analogously to Lemma 1 we have

$$
\begin{aligned}
\left\|\beta_{0}\right\| \leq & l^{\prime}(Q)\left[\Gamma+n_{2}(Q) a+l(Q)\left(1+n_{1}(Q)\right.\right. \\
& \left.\left.+Q_{1}+Q_{2} n_{1}(Q)\right) Q a+\sigma_{1} Q+\sigma_{0} Q\right]\left\|z-z^{\prime}\right\|_{I_{a}}, \\
\left\|\beta_{1}\right\| \leq & \left(1+\sigma_{2}\right) L_{2 a}\left\|z-z^{\prime}\right\|_{I_{a}}+\left(1+\sigma_{2}\right)\left[\Lambda+L_{2 a}\left(1+Q_{2}\right)\right]\left\|h-h^{\prime}\right\|_{\Delta_{a}}, \\
\left\|\beta_{2}\right\| \leq & \left(1+\sigma_{2}\right)\left[l(Q)\left(Q_{1}+Q_{2} n_{1}(Q)\right) a\right. \\
& \left.+l(Q)\left(1+n_{1}(Q)+Q_{1}+Q_{2} n_{1}(Q)\right) a+l(Q) Q+\sigma_{1}\right]\left\|z-z^{\prime}\right\|_{I_{a}} \\
& +\left(1+\sigma_{2}\right)\left[l(Q)\left(1+Q_{2}\right)\left(Q_{1}+Q_{2} n(Q)\right) a\right. \\
& \left.+l(Q)\left(1+n_{1}(Q)+Q_{1}+Q_{2} n_{1}(Q)\right) a+\sigma_{1} Q\right]\left\|h-h^{\prime}\right\|_{\Delta_{a}}, \\
\left\|\beta_{3}\right\| \leq & \left(1+\sigma_{2}\right) \sigma_{0}\left\|z-z^{\prime}\right\|_{I_{a}}+\left(1+\sigma_{2}\right)\left[G Q+\sigma_{0} Q_{2}\right]\left\|h-h^{\prime}\right\|_{\Delta_{a}} .
\end{aligned}
$$

From the above estimates we get (16). In a similar way we derive (17), which completes the proof.

\section{The main theorem}

THEOREM. Suppose that Assumptions $\mathrm{H}_{1}-\mathrm{H}_{4}$, conditions (8)-(10) and the inequality

$$
l^{\prime}(Q)\left[\Gamma+Q\left(\sigma_{1}+\sigma_{0}\right)\right]+\left(1+\sigma_{2}\right) l(Q) Q+\left(1+\sigma_{2}\right)\left(\sigma_{1}+\sigma_{0}\right)<\bar{k},
$$

are satisfied, where $\bar{k} \in(0,1)$ is a constant such that $\bar{k}>\left(\sigma_{1}+\sigma_{0}\right)\left(1+\sigma_{2}\right)$. Then for $a \in\left(0, a_{0}\right]$ sufficiently small and for any system of numbers $a_{l} \in$ $[0, a], l=1, \ldots, N$, there is a function $z \in B_{1}\left(a, Q, Q_{1}, Q_{2}\right)$ which is a 
unique solution of the problem (1), (2) in the class $B_{1}\left(a, Q, Q_{1}, Q_{2}\right)$. Furthermore, if $z, z^{\prime}$ are solutions of (1), (2) with functions $\psi, \psi^{\prime}$ respectively then

$$
\left\|z-z^{\prime}\right\|_{I_{a}} \leq(1-\bar{k})^{-1}\left(1+\sigma_{2}\right)\left\|\psi-\psi^{\prime}\right\|_{0},
$$

where $\left\|\psi-\psi^{\prime}\right\|_{0}=\sup \left\{\left\|\psi(y)-\psi^{\prime}(y)\right\|: y \in \mathbb{R}^{r}\right\}$.

Proof. Let $V=\left(V^{(1)}, V^{(2)}\right)$, where $V^{(1)}, V^{(2)}$ are defined by (6), (7). From Lemmas 1-3 it follows that

$$
\begin{aligned}
V: B_{1}\left(a, Q, Q_{1}, Q_{2}\right) \times B_{2}(a, & \left.Q, Q_{1}, Q_{2}, p\right) \\
& \rightarrow B_{1}\left(a, Q, Q_{1}, Q_{2}\right) \times B_{2}\left(a, Q, Q_{1}, Q_{2}, p\right)
\end{aligned}
$$

is continuous provided that $a$ is so small that (11), (13) and (15) hold. By (18) we may additionally assume that $a$ is small enough that

(20) $E_{1 a}<\bar{k}, \quad L_{1 a}\left(1+Q_{2}\right)<\bar{k}, \quad E_{2 a} L_{1 a}<\left(\bar{k}-E_{1 a}\right)\left(\bar{k}-L_{a}\left(1+Q_{2}\right)\right)$. Let $\alpha, \beta>0$ satisfy $L_{1 a}\left(\bar{k}-E_{1 a}\right)^{-1} \leq \alpha / \beta \leq\left(\bar{k}-L_{1 a}\left(1+Q_{2}\right)\right) E_{2 a}^{-1}$. Analogously to [4] we define in $B_{1}\left(a, Q, Q_{1}, Q_{2}\right) \times B_{2}\left(a, Q, Q_{1}, Q_{2}, p\right)$ the following weighted norm:

$$
\|w\|_{*}=\alpha\|z\|_{I_{a}}+\beta\|h\|_{\Delta_{a}}, \quad w=(z, h),
$$

where $z \in B_{1}\left(a, Q, Q_{1}, Q_{2}\right), h \in B_{2}\left(a, Q, Q_{1}, Q_{2}, p\right)$. It is easy to check (cf. [4]) that $V$ is a contraction with constant $\bar{k}$ with respect to this norm. Thus $V$ has a unique fixed point $\bar{w}=V \bar{w}, \bar{w}=(\bar{z}, \bar{h}) \in B_{1}\left(a, Q, Q_{1}, Q_{2}\right) \times$ $B_{2}\left(a, Q, Q_{1}, Q_{2}, p\right)$. Let us prove that $\bar{z}$ is a solution of (1), (2). From (6) by integration by parts we obtain

(22) $0=A^{-1}\left(x, y, \bar{z}_{(x, y)}\right)\left[\Delta_{1}(x, y)+\widetilde{\Delta}_{2}(x, y)+\Delta_{3}(x, y)\right], \quad(x, y) \in I_{a}$,

where $\Delta_{1}, \Delta_{3}$ are defined by (6) with $z=\bar{z}, h=\bar{h}$ and $\widetilde{\Delta}_{2}(x, y)=$ $\left(\widetilde{\Delta}_{21}(x, y), \ldots, \widetilde{\Delta}_{2 m}(x, y)\right)$ is defined by

$$
\widetilde{\Delta}_{2 i}(x, y)=-\int_{a_{i}}^{x}\left\langle A_{i}^{*}(t, x, y), D_{t} \bar{z}_{i}^{*}(t, x, y)\right\rangle d t, \quad i=1, \ldots, m .
$$

Multiplying $(22)$ by $A\left(x, y, \bar{z}_{(x, y)}\right)$ we obtain

$$
\Delta_{1 i}(x, y)+\widetilde{\Delta}_{2 i}(x, y)+\Delta_{3 i}(x, y)=0, \quad(x, y) \in I_{a}, i=1, \ldots, m,
$$

which for $x=a_{i}$ yields that $\bar{z}$ satisfies the boundary condition (2). Thus (22) reduces to

$$
\int_{a_{i}}^{x}\left[f_{i}\left(t, \bar{g}_{i}(t, x, y), \bar{z}_{\left(t, \bar{g}_{i}(t, x, y)\right)}\right)-\left\langle A_{i}^{*}(t, x, y), D_{t} \bar{z}_{i}^{*}(t, x, y)\right\rangle\right] d t=0,
$$

$(x, y) \in I_{a}, i=1, \ldots, m$, where $\bar{g}$ is defined by (5) for $h=\bar{h}$. By the same considerations as in [6] (in particular by using the group property for $\bar{g}$ ) we 
see that $\bar{z}$ satisfies (1) a.e. in $I_{a}$. By the reverse considerations and by the uniqueness of the fixed point of $V$ we conclude that $\bar{z}$ is a unique solution of (1), (2).

It remains to prove (19). Let $w=(z, h)=V w, w^{\prime}=\left(z^{\prime}, h^{\prime}\right)=V w^{\prime}$ be the fixed points of $V=\left(V^{(1)}, V^{(2)}\right)$ defined by (6), (7) with functions $\psi, \psi^{\prime}$ respectively. We then have

$$
\begin{aligned}
\left\|z-z^{\prime}\right\|_{I_{a}} & \leq\left(1+\sigma_{2}\right)\left\|\psi-\psi^{\prime}\right\|_{0}+E_{1 a}\left\|z-z^{\prime}\right\|_{I_{a}}+E_{2 a}\left\|h-h^{\prime}\right\|_{\Delta_{a}}, \\
\left\|h-h^{\prime}\right\|_{\Delta_{a}} & \leq L_{1 a}\left\|z-z^{\prime}\right\|_{I_{a}}+L_{1 a}\left(1+Q_{2}\right)\left\|h-h^{\prime}\right\|_{\Delta_{a}} .
\end{aligned}
$$

From this we obtain $\left(1-E_{1 a}-E_{2 a} L_{1 a}\left(1-L_{1 a}\left(1+Q_{2}\right)\right)^{-1}\right)\left\|z-z^{\prime}\right\|_{I_{a}} \leq(1+$ $\left.\sigma_{2}\right)\left\|\psi-\psi^{\prime}\right\|_{0}$. By (20) we have $1-\bar{k} \leq 1-E_{1 a}-E_{2 a} L_{1 a}\left(1-L_{1 a}\left(1+Q_{2}\right)\right)^{-1}$, and from the last inequality we derive (19). This ends the proof.

5. Examples. Put $\widehat{\Omega}=\left[0, a_{0}\right] \times \mathbb{R}^{r} \times \mathbb{R}^{m} \times \mathbb{R}^{m}$.

Assumption $\mathrm{H}_{5}$. $1^{\circ} \widehat{A} \in C(\widehat{\Omega}, M(m, m))$ and there is a constant $\widehat{\nu}>0$ such that $\operatorname{det} \widehat{A}(x, y, u, v) \geq \widehat{\nu}$ for any $(x, y, u, v) \in \widehat{\Omega}$.

$2^{\circ}$ There are constants $n, l \in \mathbb{R}_{+}$such that for all $(x, y, u, v),(\bar{x}, \bar{y}, \bar{u}, \bar{v}) \in$ $\widehat{\Omega}$ we have

$$
\begin{gathered}
\|\widehat{A}(x, y, u, v)\| \leq n, \\
\|\widehat{A}(x, y, u, v)-\widehat{A}(\bar{x}, \bar{y}, \bar{u}, \bar{v})\| \leq l[|x-\bar{x}|+\|y-\bar{y}\|+\|u-\bar{u}\|+\|v-\bar{v}\|] . \\
3^{\mathrm{o}} \widehat{\varrho}(\cdot, y, u, v):\left[0, a_{0}\right] \rightarrow M(m, r), \widehat{f}(\cdot, y, u, v):\left[0, a_{0}\right] \rightarrow M(m, 1) \text { are }
\end{gathered}
$$
measurable for any $(y, u, v) \in \mathbb{R}^{r} \times \mathbb{R}^{m} \times \mathbb{R}^{m}$ and $\widehat{\varrho}(x, \cdot): \mathbb{R}^{r} \times \mathbb{R}^{m} \times$ $\mathbb{R}^{m} \rightarrow M(m, r), \widehat{f}(x, \cdot): \mathbb{R}^{r} \times \mathbb{R}^{m} \times \mathbb{R}^{m} \rightarrow M(m, 1)$ are continuous for a.e. $x \in\left[0, a_{0}\right]$.

$4^{\mathrm{o}}$ There are Lebesgue integrable functions $\widehat{l}_{1}, \widehat{l}_{2}:\left[0, a_{0}\right] \rightarrow \mathbb{R}_{+}$and constants $n_{1}, n_{2} \in \mathbb{R}_{+}$such that for a.e. $x \in\left[0, a_{0}\right]$ and for all $(y, u, v),(\bar{y}, \bar{u}, \bar{v}) \in$ $\mathbb{R}^{r} \times \mathbb{R}^{m} \times \mathbb{R}^{m}$ we have

$$
\begin{gathered}
\|\widehat{\varrho}(x, y, u, v)\| \leq n_{1}, \quad\|\widehat{f}(x, y, u, v)\| \leq n_{2}, \\
\|\widehat{\varrho}(x, y, u, v)-\widehat{\varrho}(x, \bar{y}, \bar{u}, \bar{v})\| \leq l_{1}(x)[\|y-\bar{y}\|+\|u-\bar{u}\|+\|v-\bar{v}\|], \\
\|\widehat{f}(x, y, u, v)-\widehat{f}(x, \bar{y}, \bar{u}, \bar{v})\| \leq l_{2}(x)[\|y-\bar{y}\|+\|u-\bar{u}\|+\|v-\bar{v}\|] .
\end{gathered}
$$

Let $\alpha, \beta, \gamma:\left[0, a_{0}\right] \times \mathbb{R}^{r} \rightarrow \mathbb{R}^{1+r}$, where $\alpha=\left(\alpha_{0}, \alpha_{1}, \ldots, \alpha_{r}\right), \beta=$ $\left(\beta_{0}, \beta_{1}, \ldots, \beta_{r}\right), \gamma=\left(\gamma_{0}, \gamma_{1}, \ldots, \gamma_{r}\right)$, be given functions such that $-b_{0} \leq$ $\alpha_{0}(x, y), \beta_{0}(x, y), \gamma_{0}(x, y) \leq x$ for all $(x, y) \in\left[0, a_{0}\right] \times \mathbb{R}^{r}$. Suppose that $\alpha$ is Lipschitz continuous on $\left[0, a_{0}\right] \times \mathbb{R}^{r}$ and that $\beta, \gamma$ are Lipschitz continuous with respect to the second variable on $\mathbb{R}^{r}$. Then, if we define

$$
\begin{aligned}
A(x, y, w) & =\widehat{A}(x, y, w(0,0), w(\alpha(x, y)-(x, y))), \\
\varrho(x, y, w) & =\widehat{\varrho}(x, y, w(0,0), w(\beta(x, y)-(x, y))), \\
f(x, y, w) & =\widehat{f}(x, y, w(0,0), w(\gamma(x, y)-(x, y))),
\end{aligned}
$$


and if Assumption $\mathrm{H}_{5}$ holds then the functions $A, \varrho, f$ satisfy Assumptions $\mathrm{H}_{1}-\mathrm{H}_{3}$ respectively. Thus the hyperbolic system with retarded argument

$$
\begin{aligned}
\sum_{j=1}^{m} \widehat{A}_{i j}(x, y, z(x, y), & z(\alpha(x, y)))\left[D_{x} z_{j}(x, y)\right. \\
& \left.+\sum_{k=1}^{r} \widehat{\varrho}(x, y, z(x, y), z(\beta(x, y))) D_{y_{k}} z_{j}(x, y)\right] \\
= & \widehat{f}(x, y, z(x, y), z(\gamma(x, y))), \quad i=1, \ldots, m,
\end{aligned}
$$

is a particular case of (1).

The differential-integral system

$$
\begin{aligned}
& \sum_{j=1}^{m} \widehat{A}_{i j}\left(x, y, z(x, y), \int_{\alpha(x, y)}^{\tilde{\alpha}(x, y)} z(t, s) K(t, s, x, y) d t d s\right)\left[D_{x} z_{j}(x, y)\right. \\
& \left.+\sum_{k=1}^{r} \widehat{\varrho}_{i k}\left(x, y, z(x, y), \int_{\beta(x, y)}^{\tilde{\beta}(x, y)} z(t, s) K_{1}(t, s, x, y) d t d s\right) D_{y_{k}} z_{j}(x, y)\right] \\
= & \widehat{f}_{i}\left(x, y, z(x, y), \int_{\gamma(x, y)}^{\tilde{\gamma}(x, y)} z(t, s) K_{2}(t, s, x, y) d t d s\right), \quad i=1, \ldots, m,
\end{aligned}
$$

where $\alpha, \widetilde{\alpha}, \beta, \widetilde{\beta}, \gamma, \widetilde{\gamma}:\left[0, a_{0}\right] \times \mathbb{R}^{r} \rightarrow \mathbb{R}^{1+r}$ and $K, K_{1}, K_{2}:\left[0, a_{0}\right] \times \mathbb{R}^{r} \times$ $\left[0, a_{0}\right] \times \mathbb{R}^{r} \rightarrow M(m, m)$, is also a particular case of (1). In this case if we make suitable assumptions on $\alpha, \widetilde{\alpha}, \beta, \widetilde{\beta}, \gamma, \widetilde{\gamma}, K, K_{1}, K_{2}$ and if Assumption $\mathrm{H}_{5}$ holds then $A(x, y, w)$ defined by

$$
A(x, y, w)=\widehat{A}\left(x, y, w(0,0), \int_{\alpha(x, y)}^{\tilde{\alpha}(x, y)} w(t-x, s-y) K(t, s, x, y) d t d s\right)
$$

satisfies Assumption $\mathrm{H}_{1}$, and $\varrho, f$ similarly defined satisfy Assumptions $\mathrm{H}_{2}$, $\mathrm{H}_{3}$ respectively.

Our last example is the system

$$
\begin{aligned}
\sum_{j=1}^{m} \widehat{A}_{i j}\left(x, y, z(x, y), z\left(\alpha\left(x, y, z_{(x, y)}\right)\right)\right)\left[D_{x} z_{j}(x, y)\right. & \\
& \left.+\sum_{k=1}^{r} \widehat{\varrho}_{i k}\left(x, y, z(x, y), z\left(\beta\left(x, y, z_{(x, y)}\right)\right)\right) D_{y_{k}} z_{j}(x, y)\right] \\
= & \widehat{f}_{i}\left(x, y, z(x, y), z\left(\gamma\left(x, y, z_{(x, y)}\right)\right)\right),
\end{aligned}
$$

$i=1, \ldots, m, \alpha, \beta, \gamma: \Omega \rightarrow \mathbb{R}^{1+r}$. 
LEMMA 4. Suppose that

$1^{\mathrm{O}}$ Assumption $\mathrm{H}_{1}$ holds;

$2^{\mathrm{o}} \alpha(\cdot, y, w), \beta(\cdot, y, w), \gamma(\cdot, y, w):\left[0, a_{0}\right] \rightarrow \mathbb{R}^{1+r}$ are measurable for any $(y, w) \in \mathbb{R}^{r} \times C\left(B, \mathbb{R}^{m}\right)$, and $\alpha(x, \cdot), \beta(x, \cdot), \gamma(x, \cdot): \mathbb{R}^{r} \times C\left(B, \mathbb{R}^{m}\right) \rightarrow \mathbb{R}^{1+r}$ are continuous for a.e. $x \in\left[0, a_{0}\right]$;

$3^{\circ}$ for any $(x, y, w) \in \Omega$ we have

$$
\alpha(x, y, w) \in B, \quad \beta(x, y, w) \in B, \quad \gamma(x, y, w) \in B ;
$$

$4^{\mathrm{o}}$ there is a nondecreasing function $D: \mathbb{R}_{+} \rightarrow \mathbb{R}_{+}$such that for all $q \in \mathbb{R}_{+},(x, y, w),(\bar{x}, \bar{y}, \bar{w}) \in\left[0, a_{0}\right] \times \mathbb{R}^{r} \times C_{0+\mathrm{L}}\left(B, \mathbb{R}^{m}, q\right)$ we have

$$
\|\alpha(x, y, w)-\alpha(\bar{x}, \bar{y}, \bar{w})\| \leq D(q)\left[|x-\bar{x}|+\|y-\bar{y}\|+\|w-\bar{w}\|_{0}\right] ;
$$

$5^{\mathrm{O}}$ there are nondecreasing functions $D_{1}, D_{2}: \mathbb{R}_{+} \rightarrow \mathbb{R}_{+}$such that for all $q \in \mathbb{R}_{+}$and $(y, w),(\bar{y}, \bar{w}) \in \mathbb{R}^{r} \times C_{0+\mathrm{L}}\left(B, \mathbb{R}^{m}, q\right)$ and for a.e. $x \in\left[0, a_{0}\right]$ we have

$$
\begin{aligned}
&\|\beta(x, y, w)-\beta(x, \bar{y}, \bar{w})\| \leq D_{1}(q)\left[\|y-\bar{y}\|+\|w-\bar{w}\|_{0}\right], \\
&\|\gamma(x, y, w)-\gamma(x, \bar{y}, \bar{w})\| \leq D_{2}(q)\left[\|y-\bar{y}\|+\|w-\bar{w}\|_{0}\right] .
\end{aligned}
$$

Then the functions $A, \varrho, f$ defined by

$$
\begin{aligned}
A(x, y, w) & =\widehat{A}(x, y, w(0,0), w(\alpha(x, y, w)-(x, y))), \\
\varrho(x, y, w) & =\widehat{\varrho}(x, y, w(0,0), w(\beta(x, y, w)-(x, y))), \\
f(x, y, w) & =\widehat{f}(x, y, w(0,0), w(\gamma(x, y, w)-(x, y))),
\end{aligned}
$$

satisfy Assumptions $\mathrm{H}_{1}, \mathrm{H}_{2}, \mathrm{H}_{3}$ respectively.

Remark 3. Systems (23), (24) can also be obtained from the theory of differential-functional equations with operators of Volterra type [19]-[22]. System (25) cannot be obtained from that theory.

\section{References}

[1] P. Bassanini, On a recent proof concerning a boundary value problem for quasilinear hyperbolic systems in the Schauder canonic form, Boll. Un. Mat. Ital. (5) 14-A (1977), 325-332.

[2] —, Iterative methods for quasilinear hyperbolic systems, ibid. (6) 1-B (1982), 225250.

[3] -, The problem of Graff-Cesari, in: Nonlinear Phenomena in Math. Sci., V. Lakshmikantham (ed.), Proc. Arlington 1980, Academic Press, 1982, 87-101.

[4] P. Bassanini e E. Filliaggi, Schemi iterativi a accelerazione della convergenza per operatori di contrazione nel prodotto di due spazi di Banach, Atti Sem. Mat. Fis. Modena 28 (1979), 249-279.

[5] L. Cesari, A boundary value problem for quasilinear hyperbolic systems, Riv. Mat. Univ. Parma 3 (1974), 107-131. 
[6] L. Cesari, A boundary value problem for quasilinear hyperbolic systems in the Schauder canonic form, Ann. Scuola Norm. Sup. Pisa (4) 1 (1974), 311-358.

[7] T. Człapiński, On the Cauchy problem for quasilinear hyperbolic systems of partial differential-functional equations of the first order, Z. Anal. Anwendungen 10 (1991), 169-182.

[8] -, A boundary value problem for quasilinear hyperbolic systems of partial differential-functional equations of the first order, Boll. Un. Mat. Ital. (7) 5-B (1991), 619637.

[9] T. Człapiński and Z. Kamont, Generalized solutions of quasi-linear hyperbolic systems of partial differential-functional equations, to appear.

[10] J. Hale, Functional Differential Equations, Springer, New York 1971.

[11] Z. Kamont, Existence of solutions of first order partial differential-functional equations, Comment. Math. 25 (1985), 249-263.

[12] Z. Kamont and J. Turo, On the Cauchy problem for quasilinear hyperbolic system of partial differential equations with a retarded argument, Boll. Un. Mat. Ital. (6) 4-B (1985), 901-916.

[13] - - - On the Cauchy problem for quasilinear hyperbolic systems with a retarded argument, Ann. Mat. Pura Appl. 143 (1986), 235-246.

[14] —, - A boundary value problem for quasilinear hyperbolic systems with a retarded argument, Ann. Polon. Math. 47 (1987), 347-360.

[15] - - - Generalized solutions of boundary value problems for quasilinear systems with retarded argument, Radovi Mat. 4 (1988), 239-260.

[16] V. Lakshmikantham and S. Leela, Differential and Integral Inequalities, Vol. 2, Academic Press, New York 1969.

[17] N. Mattioli and M. C. Salvatori, A theorem of existence and uniqueness in nonlinear dispersive optics, Atti Sem. Mat. Fis. Univ. Modena 28 (1979), 405-424.

[18] A. Salvadori, Sul problema di Cauchy per una struttura ereditaria di tipo iperbolico. Esistenza, unicità e dipendenza continua, ibid. 32 (1983), 329-356.

[19] J. Turo, A boundary value problem for quasilinear hyperbolic systems of hereditary partial differential equations, ibid. 34 (1985-86), 15-34.

[20] - On some class of quasilinear hyperbolic systems of partial differential-functional equations of the first order, Czechoslovak Math. J. 36 (111) (1986), 185-197.

[21] - Existence and uniqueness of solutions of quasilinear hyperbolic systems of partial differential-functional equations, Math. Slovaca 37 (1987), 375-387.

[22] —, A boundary value problem for hyperbolic systems of differential-functional equations, Nonlinear Anal. 13 (1) (1989), 7-18.

INSTITUTE OF MATHEMATICS

UNIVERSITY OF GDAŃSK

WITA STWOSZA 57

80-952 GDAŃSK, POLAND

Reçu par la Rédaction le 4.11.1991

Révisé le 15.2.1992 